\title{
The colours of Virgo dEs as seen by SDSS
}

\author{
Thorsten Lisker, Eva K. Grebel and Bruno Binggeli
}

Astronomical Institute, University of Basel, Switzerland; email: tlisker@astro.unibas.ch

\begin{abstract}
A colour analysis of a carefully selected sample of over 200 Virgo cluster dEs and 8 giant ellipticals (Es) in the SDSS yields the following results: a) the nucleated dEs (dENs) follow a tight colour-magnitude relation (CMR) which does not broaden significantly at fainter $\left(M_{r} \geqslant-15\right)$ magnitudes, b) the CMR of dENs smoothly transitions to the CMR of Es, but the latter changes its slope at about $M_{r} \approx-20$, c) the brighter dENs are consistent with ages of $t \geqslant 5 \mathrm{Gyr}$ and a decrease of metallicity towards fainter magnitudes, but a possible additional luminosity-age relation is not ruled out, d) it is crucial to treat dS0s separately from dEs since their different colour properties would otherwise bias the comparison of nucleated and nonnucleated dEs (dEnoNs), e) the dEnoNs show a weak trend towards bluer colours than dENs.
\end{abstract}

Keywords. galaxies: dwarf, galaxies: clusters: general, galaxies: fundamental parameters

\section{Introduction and sample selection}

Numerous studies of dwarf ellipticals (dEs) have put the homogeneity of this class of galaxy in question. In particular, the two main subclasses, nucleated and non-nucleated dEs (dENs and dEnoNs), might have a different history: dENs are more strongly clustered, are brighter, and have been claimed to show redder colours (Binggeli \& Cameron 1991; Rakos et al. 2004). To explore the differences and similarities of these galaxies in greater detail, we study here the fundamental relation of dEs - the colour-magnitude relation $(\mathrm{CMR})$ - separately for dENs and dEnoNs. This requires a large and homogeneous dE sample which ideally should also enable us to address the issue of a possible transition from dwarf to giant ellipticals (Es). Contrary to the earlier belief, it has been shown recently that the structural parameters of dEs and Es exhibit a smooth transition (Graham \& Guzman 2003), raising the question whether this is also the case for their colour properties.

The SDSS Data Release 3 provides reduced and calibrated images in the $\mathrm{u}, \mathrm{g}, \mathrm{r}, \mathrm{i}$, and $\mathrm{z}$ band as well as photometric measurements for Virgo cluster galaxies. However, low surface brightness objects like the dEs can be 'shredded', i.e. parts of them are assigned to multiple separate source detections by the detection algorithm, resulting in unreliable photometry. A comparison with the work of Gavazzi et al. (2005) showed that we must restrict ourselves to dEs brighter than $m_{r} \lesssim 18$ that are already listed in the Virgo Cluster Catalog (VCC; Binggeli et al. 1985) and carefully exclude misaligned or shredded source detections visually. Moreover, the SDSS photometric pipeline overestimates the local sky flux due to the large apparent object sizes, for which we apply an appropriate correction. Our final sample comprises $220 \mathrm{dEs}+\mathrm{dS} 0 \mathrm{~s}$; the sample of 8 giant ellipticals was selected in the same manner. Colours for the dwarfs were derived from aperture photometry on the SDSS images, excluding the inner $r \leqslant 2^{\prime \prime}$ to avoid nucleus light entering the aperture. Since this does not require any photometric measurements from the SDSS pipeline, our sample is extended to $349 \mathrm{dEs}+\mathrm{dSO}$ s in diagrams where only colours are required. Errors were estimated from the signal-to-noise ratio and the uncertainty of the sky flux correction. Galactic extinction corrections are provided by the SDSS database. 
Colour-magnitude relation: nucleated $(\bullet)$ \& non-nucl. $(x)$ dEs

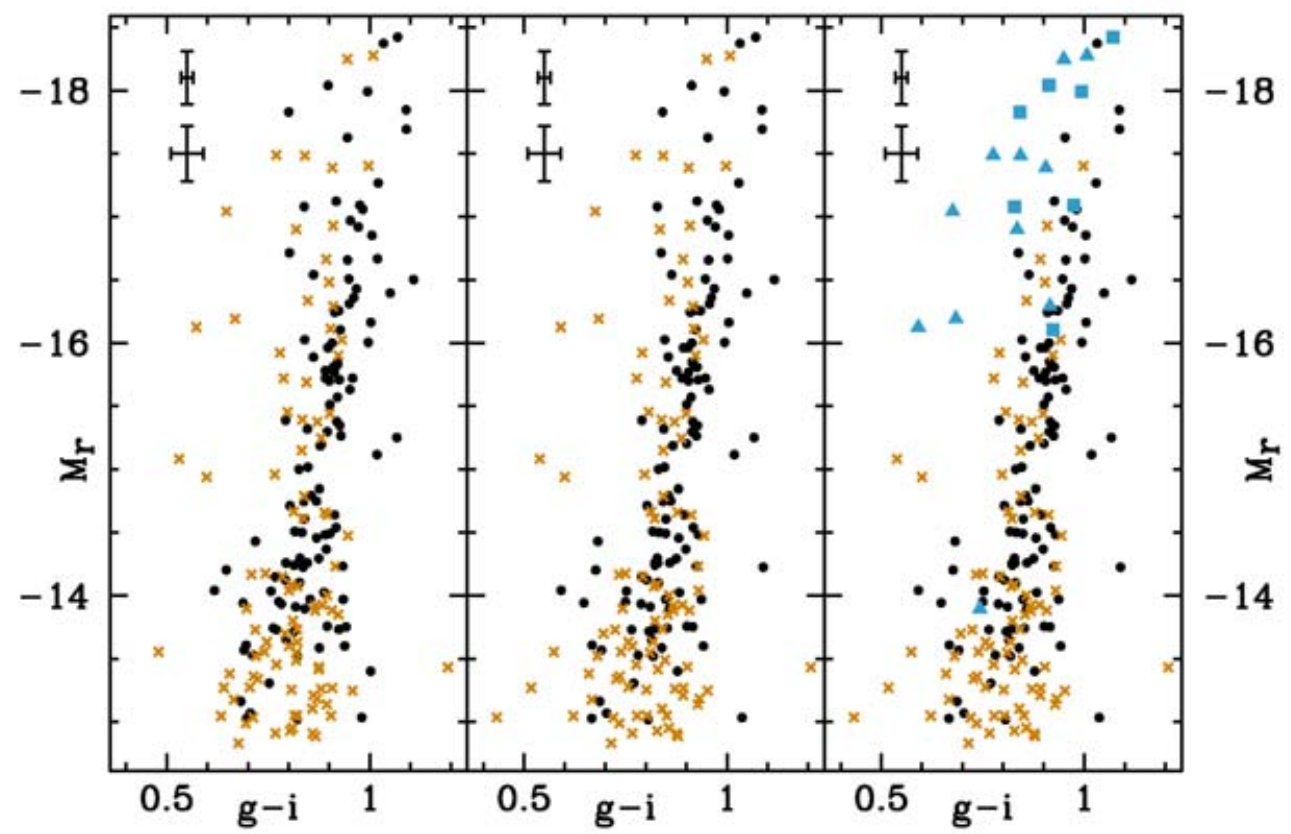

Figure 1. CMR for dENs (filled circles), dEnoNs (crosses), nucleated dS0s (squares) and non-nucleated dS0s (triangles). Upper and lower error bars indicate mean magnitude uncertainties for $M_{r} \lesssim-15.5$ and $M_{r} \gtrsim-15.5$, respectively. A distance modulus of $m-M=31$ is adopted. Left: Absolute r-band magnitude versus fixed aperture $\left(r \approx 8^{\prime \prime}\right) g-i$ colour, including dS0s into the dE class. Middle: CMR with $g-i$ colour measured from half-light radius apertures, including dS0s into the dE class. Right: CMR with $g-i$ colour measured from half-light radius apertures, now showing dS0s separately.

\section{Results}

The classification of several dwarfs as dS0 in the VCC had been based on the twodimensional appearance of the galaxies rather than on their azimuthally averaged profiles - the latter are indistinguishable from those of the dEs (Binggeli \& Cameron 1991). Consequently, the existence of a separate class of dS0 galaxies has been questioned: Ryden et al. (1999) conclude that there are no compelling arguments for it, and e.g. Barazza et al. (2003) subsume dEs and dS0s into one class. We demonstrate the danger of this approach in Fig. 1, where we show the CMR separately for dENs and dEnoNs, treating dSOs as dEs in the middle panel but separating them in the right panel. From the middle panel one would conclude that the CMR of dEnoNs shows a steeper slope than the one of dENs, whereas the right panel reveals only a slight tendency towards bluer colours of dEnoNs compared to dENs, also given the paucity of bright dEnoNs. Here and in the following figures colour is measured within the half-light radius; the left panel of Fig. 1 confirms that no significant difference appears when using fixed apertures instead. The CMR of dENs is tight and does not broaden towards fainter magnitudes, as opposed to the findings of previous studies of dEs (e.g. Conselice et al. 2003). Note that the increase in colour errors by more than a factor of two would require any significant broadening to be larger than that, which is not the case.

We extend the CMR to faint Es in Fig. 2, illustrating that there is a smooth transition from dEs to Es which is mainly populated by dENs. The left panel shows the positions of 


\section{Colour-magnitude relation: from dwarfs to giants}

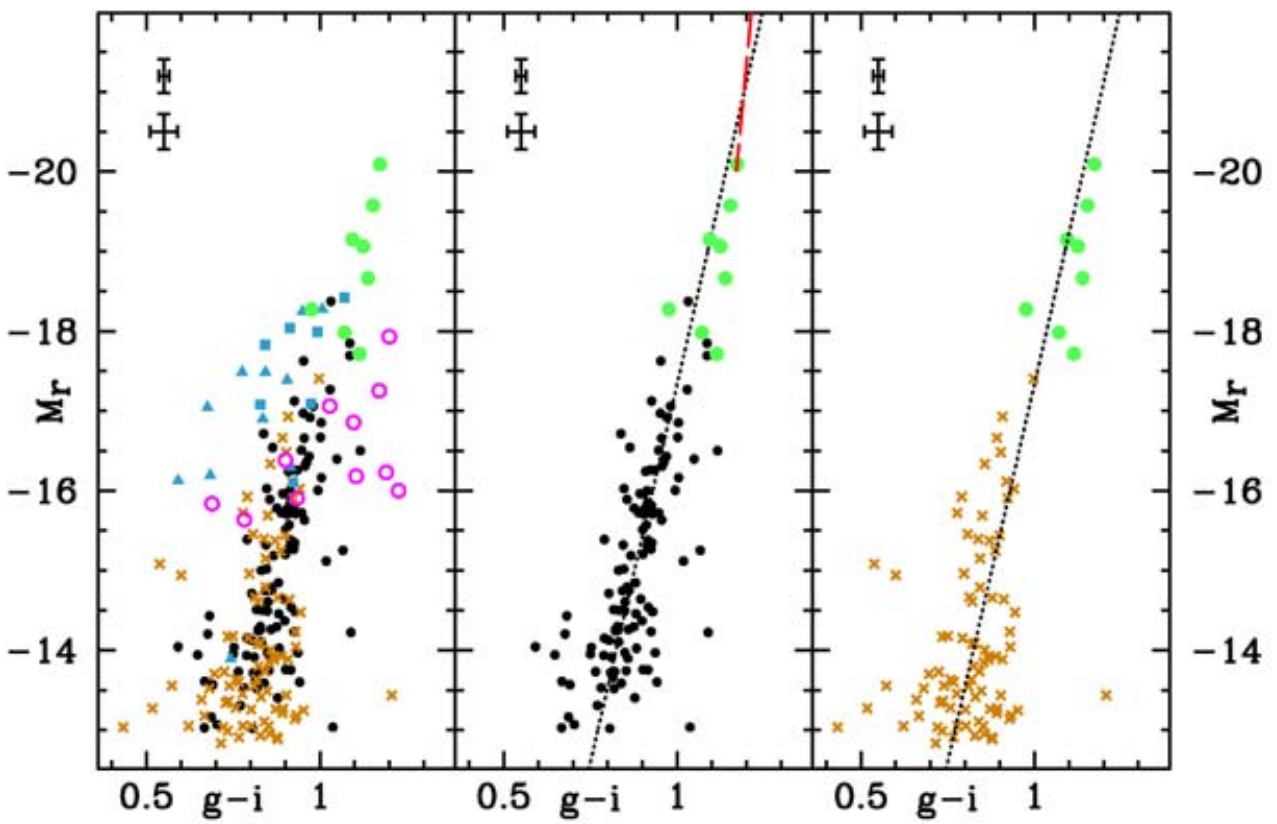

Figure 2. Left: CMR for dENs (filled circles), dEnoNs (crosses), nucleated dS0s (squares), non-nucleated dS0s (triangles), compact faint (M32-like) ellipticals (open circles), and Es (large filled circles). Middle: CMR for dENs and Es only. The dotted line shows a least-squares fit to the dENs, the dashed line is the slope of the CMR of brighter giant ellipticals from Chang et al. 2005. Right: CMR for dEnoNs and Es only. The dotted line is the same as in the middle panel.

various types of galaxy and demonstrates that compact faint 'M32-like' ellipticals neither belong to the E nor to the dE class but define their own colour-magnitude region instead. In the middle panel we show that the Es basically follow the linear extension of the CMR of dENs as derived from a least-squares fit. However, a study of nearby Es in the SDSS by Chang et al. (2005) finds a steeper slope which is well constrained for magnitudes $M_{r} \lesssim-20$ and indicates a slope change at about this magnitude. In the right panel we focus on the CMR of dEnoNs in order to see whether or not they follow the relation defined by dENs and Es. Unfortunately the small number of bright dEnoNs prevents any definite conclusion - despite a weak tendency towards bluer colours the dEnoNs are not inconsistent with the slope defined by the dENs.

A rough age and metallicity distribution of our sample can be obtained through a multicolour comparison of the observations with population synthesis models from Bruzual \& Charlot (2003), as shown in Fig. 3. Since the range of values for $i-z$ is rather small, we select only objects brighter than $m_{r} \leqslant 16$ to avoid large errors that would dilute the comparison. Brighter objects tend to be redder because of the CMR (left panel of Fig. 3), therefore the colours can only be explained with a decrease in metallicity towards fainter magnitudes (right panel), consistent with previous studies. Young ages $(t \lesssim 2 \mathrm{Gyr})$ are clearly ruled out, and for the dENs the data is consistent with ages $t \gtrsim 5$ Gyr. However, we cannot exclude a possible relation of age and luminosity on top of the dominant luminosity-metallicity relation; moreover, the trend of dEnoNs showing bluer colours appears to be strongest in $u-g$, possibly indicating younger ages than for the dENs. 
Observational vs. model colours: nucl. (•) \& non-nucl. $(x)$ dEs

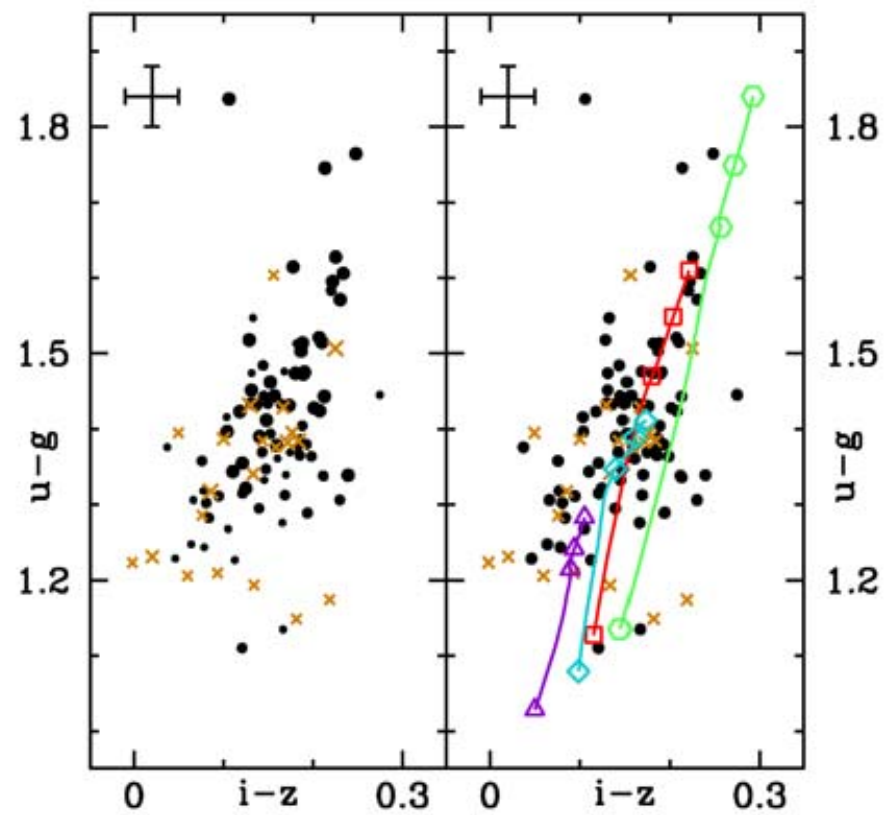

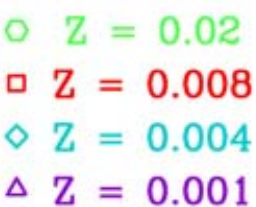

Age steps:

14..10..6..2 Gyr

(top to bottom)

Exp. decay:

$\tau=0.5 \mathrm{Gyr}$

Figure 3. Colour-colour plot for dENs (filled circles) and dEnoNs (crosses). Only objects brighter than $m_{r} \leqslant 16$ were selected to ensure small errors in the colour. Left: The effect of the CMR is illustrated by the symbol sizes: larger for brighter objects. Right: Comparison of observed and model colours. Each track from Bruzual \& Charlot (2003) shows the colours of a single-burst stellar population with a decay time of $\tau=0.5 \mathrm{Gyr}$ and an age of 2 (bottom) to 14 (top) Gyr using Padova 2000 isochrones and a Chabrier IMF.

\section{Conclusions}

Our large sample of Virgo cluster dEs+dS0s from the SDSS shows that dENs follow a tight CMR down to $M_{r} \approx-13$ without any significant broadening in colour. The faint Es follow the CMR defined by dENs, but a change in slope occurs at $M_{r} \approx-20$. After excluding dS0 galaxies, only a weak trend towards bluer colours of dEnoNs compared to $\mathrm{dENs}$ is found. The colours of the brighter dEs rule out young ages $(t \lesssim 2 \mathrm{Gyr})$ and the dENs are consistent with ages $t \gtrsim 5$ Gyr.

\section{Acknowledgements}

We thank the SDSS collaboration for the wealth of DR3 data. The SDSS Web site is http://www.sdss.org/.

\section{References}

Barazza, F.D., Binggeli, B. \& Jerjen, H. 2003, A\&A 407, 121

Binggeli, B., Sandage, A. \& Tammann, G.A. 1985, AJ 90, 1681

Binggeli, B. \& Cameron, L.M. 1991, A\& A 252, 27

Bruzual, G. \& Charlot, S. 2003, MNRAS 344, 1000

Chang, R., Gallazzi, A., Kauffmann, G. et al. 2005, astro-ph/0502117

Gavazzi, G., Donati, A., Cucciati, O. et al. 2005, A\&A 430, 411

Graham, A.W. \& Guzmán, R. 2003, AJ 125, 2936

Rakos, K. \& Schombert, J. 2004, AJ 127, 1502

Ryden, B.S., Terndrup, D.M., Pogge, R.W. \& Lauer, T.R. 1999, ApJ 517, 650 


\section{Discussion}

FERGuson: Quite a few galaxies in your colour-colour diagram were inconsistent with any of the models. Are you confident that there are not systematic uncertainties in the models or data? How far do you think you could shift the model colours, e.g. using different isochrones?

LISKER: I was using Padova 2000 isochrones against the recommendation of Bruzual \& Charlot (2003), since the Padova 1994 isochrones were offset towards redder $i-z$ colours by a noticeable amount - so indeed, we are facing a 'model error' in addition to the observational error.

RAKOs: Would you agree that the difference in the slope you have shown between $\mathrm{E}$ and $\mathrm{dE}$ galaxies is introduced by different metallicity?

LISKER: Since both the shallower slope of the CMR at $M_{r} \lesssim-20$ and the steeper slope at $M_{r} \gtrsim-20$ are luminosity-metallicity relations, I am not sure whether the change in the slope can be caused by metallicity itself.

Conselice: If you were to include all of the fainter dEs from the VCC that are not included in your sample, you would probably see a larger scatter in the CMR at faint magnitudes.

LISKER: We cannot test this so far, since the SDSS photometry catalogs are not useful for the faintest dEs, mainly due to galaxy shredding. However, we already go down to $M_{r} \approx-13$, clearly fainter than where a significant increase in the colour spread has been claimed to appear.

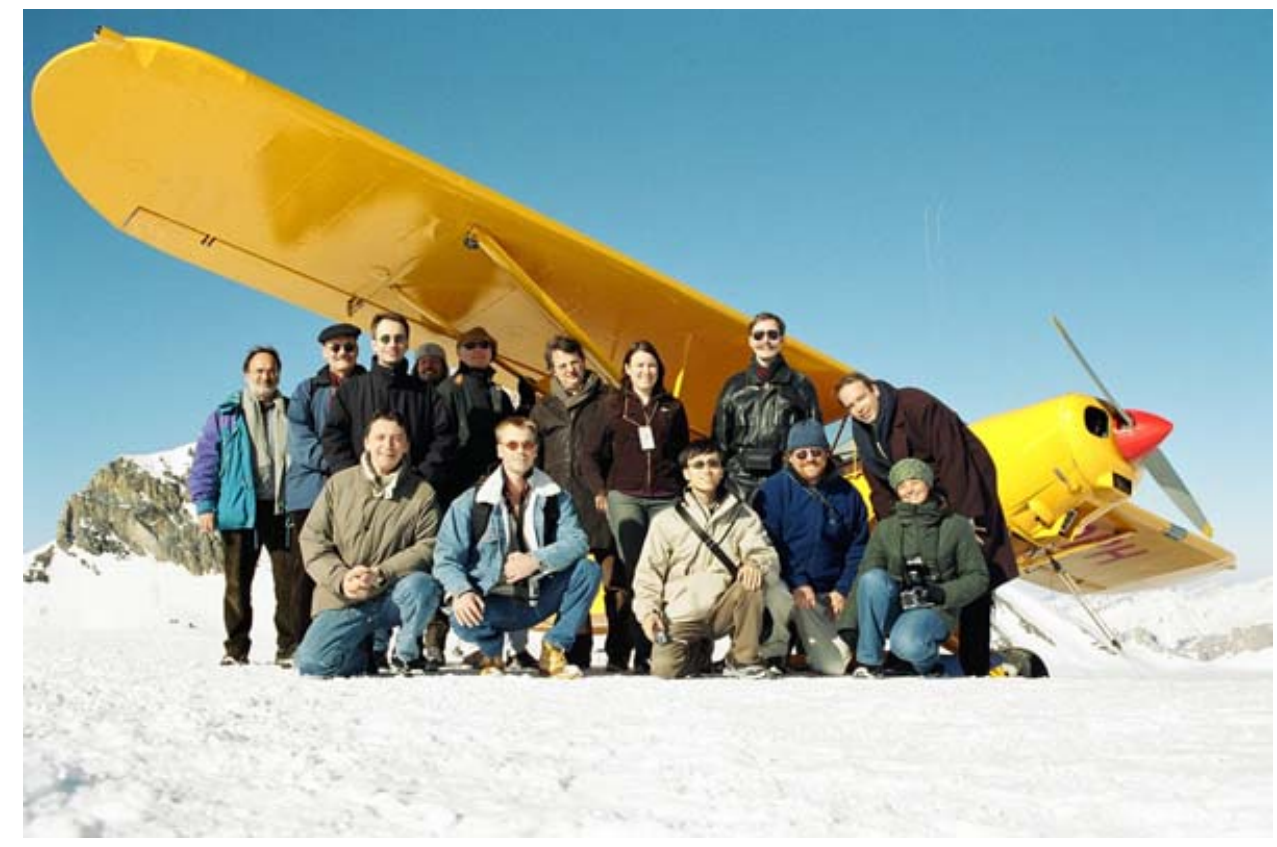

